\title{
Ultrasound Diagnosis of Cervical Vagal Schwannoma Misdiagnosed as Nodular Goiter
}

\author{
Ieong Chon Man, Kong Soi Chau \\ Department of Radiology, Conde de São Januário Hospital, Macao, China \\ Email: benjaminicm@yahoo.com.hk
}

How to cite this paper: Man, I.C. and Chau, K.S. (2018) Ultrasound Diagnosis of Cervical Vagal Schwannoma Misdiagnosed as Nodular Goiter. Open Journal of Radiology, 8, 203-208.

https://doi.org/10.4236/ojrad.2018.84023

Received: September 23, 2018

Accepted: October 16, 2018

Published: October 19, 2018

Copyright () 2018 by authors and Scientific Research Publishing Inc. This work is licensed under the Creative Commons Attribution International License (CC BY 4.0).

http://creativecommons.org/licenses/by/4.0/

\begin{abstract}
Background: Schwannoma is a benign tumor derived from Schwann cells. The most common location was cerebellopontine angle (CPA). Neurilemmoma originated from the thyroid gland is very rare. Purpose: To discuss the diagnosis and treatment of cervical vagal schwannoma and the causes of misdiagnosis and preventive measures. Case Presentation: A case of cervical vagal schwannoma misdiagnosed as nodular goiter by ultrasonography was analyzed retrospectively. This patient was found to have pain in the neck for 1 month and then went to our hospital for treatment. After admission, a $5 \mathrm{~cm} \times$ $4 \mathrm{~cm}$ mass was found on the left side of the neck, with a medium texture and clear margin. It could move with swallowing. Initially ultrasound showed a well circumscribed hypoechoic mass in the left thyroid lobe, which is suggestive of hemorrhage of thyroid nodule. Biopsy of thyroid nodules after ultrasound guided biopsy revealed Schwannoma. Surgical treatment and postoperative pathological examination confirmed cervical vagal schwannoma. The patient recovered well and was discharged 9 days after operation. Conclusion: The location of thyroid schwannoma is rare, the relationship between thyroid schwannoma and surrounding tissues is unclear, and there is no typical ultrasonic manifestation. Moreover, if doctors are not aware of their knowledge, it is easy to cause misdiagnosis. Radiologists should raise awareness of the disease and carefully analyze the results of ultrasonography in combination with the clinical manifestations of the patients so as to reduce or avoid misdiagnosis of cervical schwannoma.
\end{abstract}

\section{Keywords}

Schwannoma, Head and Neck Neoplasms, Ultrasonography, Misdiagnosis, Goiter, Nodular

\section{Introduction}

Schwannoma, also known as Schwann cell tumor, is a benign tumor originating 
from the nerve sheath. Those in the head and neck accounted for $25 \%$ to $45 \%$ of the schwannoma [1]. The main sources were sympathetic nerve (26.9\%), brachial plexus (23.6\%), vagus nerve (21.8\%), cervical plexus (9.8\%) and hypoglossal nerve (3.6\%) [2]. Schwannomas of the head and neck may present as nodules of the thyroid, regardless of the presence of cervical or (rarely) intrathyroid lesions [3] [4] [5]. The occurrence of thyroid is extremely rare, and there are few reported cases. Recently, a patient with cervical schwannoma was admitted to our hospital. In the medical system of Macau, this case is reported because the disease is rare and no similar cases have been reported. The report is as follows. This case report was approved by our hospital ethics committee.

\section{Case Presentation}

A 21-year-old male presented with our hospital with a 1-month history neck swelling and pain. He had no previous radiological examination, denied any history of head and neck radiation, and no family history of thyroid disease. The patient also denied any symptoms of hypo- or hyperthyroidism. His physical exam showed an enlarged lump on the left thyroid region and the mass can move with swallowing. Laboratory showed normal thyroid function test. On imaging, neck ultrasound showed a well circumscribed hypoechoic mass without calcification, the size was about $4.5 \times 5 \mathrm{~cm}$ in dimension and the mass is not separable from thyroid tissue (Figure 1). Then fine needle aspiration (FNA) was performed under ultrasound guidance. The pathological result of FNA showed suggestive of neural sheath tumor. Computed tomography was performed that showed a $4.5 \mathrm{~cm} \times 6.0 \mathrm{~cm} \times 4.3 \mathrm{~cm}$, fusiform hypodense mass found in the left lower cervical region, it is well-demarcated with adjacent structures; the left thyroid lobe, esophagus, trachea, the left common carotid artery and left jugular vein are compressed by the mass (Figure 2).

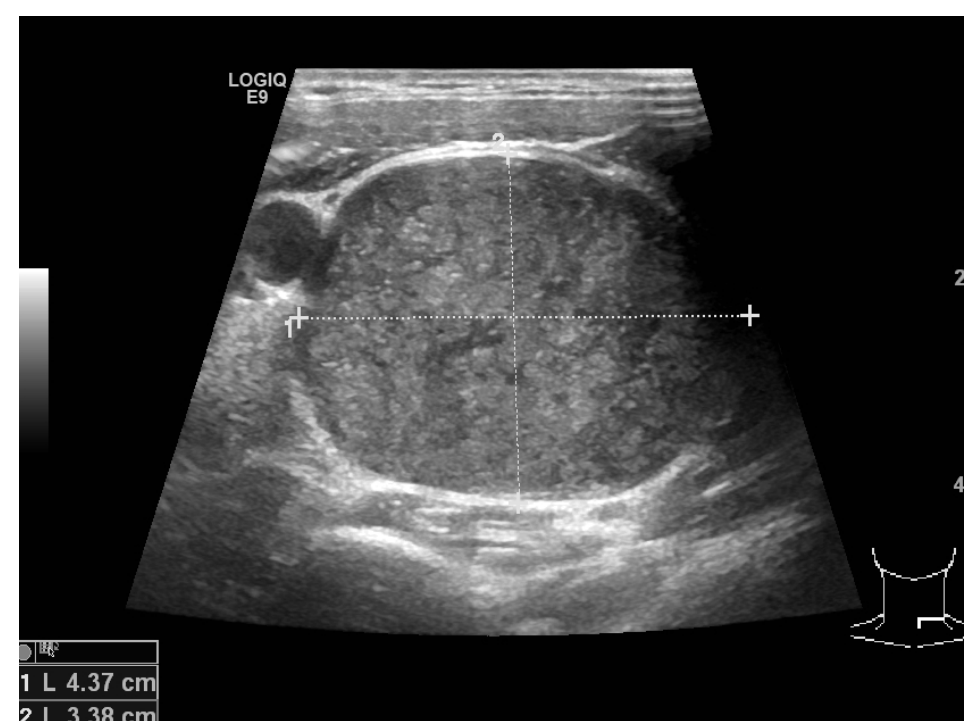

Figure 1. Transverse ultrasound image. A well circumscribed hypoechoic image, which is intrinsic with the left thyroid lobe. 


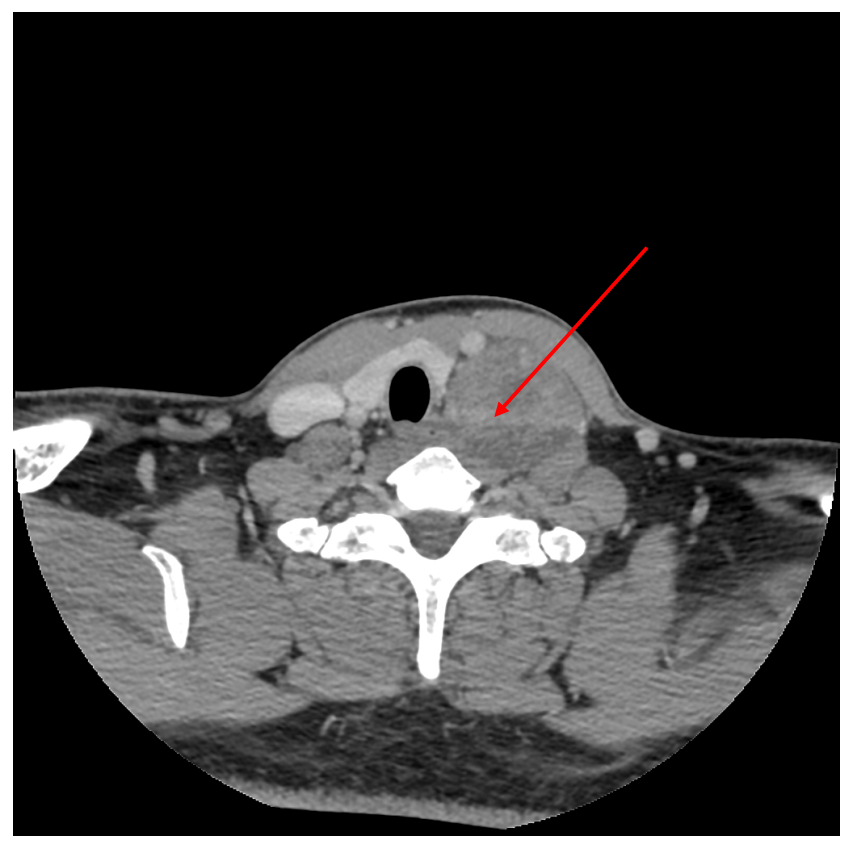

Figure 2. A $4.5 \mathrm{~cm} \times 6.0 \mathrm{~cm} \times 4.3 \mathrm{~cm}$, fusiform hypodense mass found in the left lower cervical region, it is well-demarcated with adjacent structures; the left thyroid lobe, esophagus, trachea, the left common carotid artery and left jugular vein are compressed by the mass.

Intraoperatively, the tumor was between the jugular vein and the carotid artery, deeply relation with the cervical spine, probably the mass is originated from a branch of the vagus of the Frenic nerves. The mass was removed completely and sent for pathological examination. The final pathology confirmed the diagnosis of schwannoma. The wound healed well after operation. No recurrence was observed by ultrasonography.

\section{Discussion}

Schwannoma is a benign tumor originated from peripheral nerve Schwan cells [6] [7]. The etiology of primary schwannoma is not yet clear. Its pathogenesis may be related to neurofibromatosis-2 (NF-2) gene inactivation [8]. Some authors believe that schwannomas may originate from the internal thyroid nerves [6] [9] [10]. Schwannoma grew slowly. Most of them originated from cervical plexus, cervical sympathetic nerve and vagus nerve in head and neck. The most common sites of head and neck were parapharyngeal space and cervical lateral area. In General, the neck mass is clear and generally without obvious symptoms. However, some cases have been reported with clinical manifestations of pain, dysphagia, dysphagia, facial erythema and dysphonia [11] [12]. It is reported that schwannoma in the head and neck accounts for $25 \%$ to $45 \%$ of all schwannoma. Most of the schwannomas occur at the age of $20-40$ [13]. There is no difference in gender [14] [15].

The diagnosis of schwannoma is mainly based on clinical manifestations and imaging examinations. Pathological examination is the final diagnosis [16]. 
Imaging examinations of schwannoma include ultrasound, CT and MRI. Ultrasound is a non-invasive and radiative examination. Therefore, it is mostly used in the nature of tumor, which is solid or cystic. Most of the ultrasonographic findings were hypoechoic and clearly defined masses. But the location is hard to ascertain. It is often mistaken for thyroid or salivary masses. The case was initially diagnosed by ultrasound and misdiagnosed as thyroid mass.

The sensitivity of CT and MRI in diagnosing schwannoma is higher than that of ultrasound. In the literature, schwannomas show diversity. It can be characterized by low density, equal density or high density, and variable Texture (inhomogeneous or homogeneous) and CT enhancement [17] [18]. Inhomogeneity is attributed to cystic degeneration, xanthoma-like changes, or relatively hypocellular areas adjacent to dense cells or collagen areas [19]. Schwannoma is a neoplasm with hypovascularity, but images are obtained within 60 seconds after injection of contrast medium. The main reason is that the venous drainage is not good, resulting in the accumulation of contrast medium, which mimics vascular excessive lesions and can show significant enhancement. MRI findings of schwannoma include low to medium signal intensity on T1WI, inhomogeneous high signal intensity on T2WI, and inhomogeneous post-gadolinium enhancement [18] [20]. In addition, there are other features, such as elliptical masses along the trunk of the nerve.

The target sign. On T1WI, the central region showed a moderate signal and a low signal on the edge. In the T2WI display center area, the signal is heterogeneous, the edge is hyperintensity, and the capsule is hyperintensity.

Fat tail sign. The intermuscular fat or nerve bundles around the upper and lower poles of the schwannoma encircled fat to form fat tail syndrome [21].

Surgical treatment is the first choice for schwannomas. After complete resection, the recurrence rate is low. Surgery should be done in order to preserve the nerve trunk and completely remove the tumor. In order to prevent recurrence, some scholars suggested that the tumor capsule should be removed simultaneously [22] [23] [24].

Thyroid schwannoma is a rare disease. It was misdiagnosed as thyroid nodule in demonstration and ultrasound examination. Better dialogue with surgeons and pathologists, using USG-guided FNAC and intraoperative frozen sections will definitely improve preoperative diagnosis. More reports will enhance our understanding and improve our diagnostic accuracy.

\section{Conflicts of Interest}

The authors declare no conflicts of interest regarding the publication of this paper.

\section{References}

[1] Grabowski, L. (2008) A Rare Case of Schwannoma of the Tongue. Otolaryngologia Polska, 62, 191-194. https://doi.org/10.1016/S0030-6657(08)70239-7

[2] Xu, B.Y., Yin, Z.W., Qian, H.B., et al. (2014) The Analysis on 275 Cases of Head and 
Neck Neurolimmoma. Chinese Journal of Clinical Oncology, 31, 29-30.

[3] Subramaniam, V. and Adarsha, T. (2010) Schwannoma of the Thyroid Gland-A Case Report. Journal De Chirurgie, 6, 536-538.

[4] Pillai, S., Agarwal, A.C., Mathew, M. and Nayak, D.R. (2013) Ancient Schwannoma Mimicking a Thyroid Mass with Retrosternal Extension. BMJ Case Reports, 3, pii: bcr2013200608. https://doi.org/10.1136/bcr-2013-200608

[5] Dhar, H., Dabholkar, J.P., Kandalkar, B.M. and Ghodke, R. (2014) Primary Thyroid Schwannoma Masquerading as a Thyroid Nodule. Journal of Surgical Case Reports, 2014, pii: rju094.

[6] Aron, M., Kapila, K. and Verma, K. (2005) Neural Tumors of the Neck Presenting as Thyroid Nodules: A Report of Three Cases. Cytopathology, 16, 206-209. https://doi.org/10.1111/j.1365-2303.2005.00246.x

[7] Coleman, B.G., Arger, P.H., Dalinka, M.K., Obringer, A.C., Raney, B.R. and Meadows, A.T. (1983) CT of Sarcormatous Degeneration in Neurofibromatosis. American Journal of Roentgenology, 140, 383-387. https://doi.org/10.2214/ajr.140.2.383

[8] Antinheimo, J., Sallinen, S.L., Sallinen, P., et al. (2000) Genetic Aberrations in Sporadic and Neurofibromatosis 2 (NF2)-Associated Schwannomas Studied by Comparative genomic Hybridization (CGH). Acta Neurochirurgica, 142, 1104-1105. https://doi.org/10.1007/s007010070036

[9] Cashman, E., Skinner, L. and Timon, C. (2008) Thyroid Swelling: An Unusual Presentation of a Cervical Sympathetic Chain Schwannoma. The Medscape Journal of Medicine, 10, 201.

[10] Hamza, A., Fagan, J.J., Weissman, J.L. and Myers, E.N. (1997) Neurilemomas of the Parapharyngeal Space. Archives of Otolaryngology_Head and Neck Surgery, 123, 622-626. https://doi.org/10.1001/archotol.1997.01900060064011

[11] Million, R.R. and Cassisi, N.J. (1994) Management of Head and Neck Cancer. 2nd Edition, Lippincott, Philadelphia, 785-810.

[12] Sagar, S.M. and Israel, M.A. (2001) Primary and Metastatic Tumors of the Nervous System. In: Braunwald, E., Fauci, A.S. and Kasper, D.L., Eds., Harrison's Principles of Internal Medicine, 15th Edition, McGraw-Hill, New York, 2442-2452.

[13] Subhashish, D., Kalyani, R. and Harendra, K.M. (2008) Vulval Schwannoma: A Cytological Diagnosis. Journal of Cytology, 25, 108-110. https://doi.org/10.4103/0970-9371.44048

[14] Zhang, X.F., Hu, Y., Xiao, S.H., et al. (2002) Operation Treatment for 543 Cases of Neurilemoma. Orthopedic J Chin, 9, 658-661.

[15] Li, J.Y. and Qi, J.P. (2014) Clinical Analysis on 459 Cases of Schwannoma Multiple Sites. Chin Prim Heal Care, 28, 120-121.

[16] Liu, H.L., Yu, S.Y., Li, K.H., et al. (2011) Extracranial Head and Neck Schwannomas: A Study of the Nerve of Origin. European Archives of Oto-Rhino-Laryngology, 268, 1343-1347. https://doi.org/10.1007/s00405-011-1491-4

[17] Silver, A.J., Mawad, M.E., Hilal, S.K., et al. (1984) Computed Tomography of the Carotid Space and Related Cervical Spaces. Part II. Neurogenic Tumors. Radiology, 150, 729-735. https://doi.org/10.1148/radiology.150.3.6695075

[18] Som, P.M., Sacher, M., Stollman, A.L., et al. (1988) Common Tumors of the Parapharyngeal Space: Refined Imaging Diagnosis. Radiology, 169, 81-85. https://doi.org/10.1148/radiology.169.1.2843942

[19] Cohen, L.M., Schwartz, A.M. and Rockoff, S.D. (1986) Benign Schwannoma: Pathological Basis for CT Inhomogeneities. American Journal of Roentgenology, 147, 
141-143. https://doi.org/10.2214/ajr.147.1.141

[20] Som, P.M. and Curtin, H.D. (1996) Parapharyngeal Space. In: Som, P.M. and Curtin, H.D., Eds., Head and Neck Imaging, Vol. 2. 3rd Edition, Mosby, St Louis, 915-951.

[21] Zhao, S.C., Zhang, C.L. and Zeng, B.F. (2012) The Clinical Features and Surgical Treatment of Peripheral Nerve Schwannomas. Chin J Bone Joint, 1, 263-267.

[22] Som, P.M., Biller, H.F., Lawson, W., et al. (1984) Parapharyngeal Space Masses: An Updated Protocol Based upon 104 Cases. Radiology, 153, 149-156. https://doi.org/10.1148/radiology.153.1.6089262

[23] Wan, B.L., Wang, L., Zhu, Y.L., et al. (2007) Clinical Analysis of 33 Cases of Neurilemmomas of the Head and Neck. Clin J Otorhinolarynol Head Neck Surg, 17, 794-795.

[24] Colreavy, M.P., Lacy, P.D., Hughes, J., et al. (2000) Head and Neck Schwannomas-A 10-Year Review. The Journal of Laryngology \& Otology, 114, 119-124. https://doi.org/10.1258/0022215001905058 